\title{
An Amplified WDM-PON Using Broadband Light Source Seeded Optical Sources and a Novel Bidirectional Reach Extender
}

\author{
Byoung-Wook Kang and Chul Han Kim* \\ School of Electrical and Computer Engineering, University of Seoul, Seoul 130-743, Korea
}

(Received April 13, 2011 : revised June 15, 2011 : accepted June 17, 2011)

\begin{abstract}
We demonstrated the feasibility of an amplified wavelength-division multiplexed passive optical network (WDM-PON) architecture based on broadband light source (BLS) seeded optical sources and a novel bidirectional reach extender. Our bidirectional reach extender could provide an amplification of both downstream and upstream signals as well as a BLS output for the upstream WDM signal generation. An error-free $1.25 \mathrm{~Gb} / \mathrm{s}$ signal transmission over a 100-km long single-mode fiber was achieved in a bidirectional WDM-PON using BLS seeded reflective semiconductor optical amplifier (RSOA) sources.
\end{abstract}

Keywords: Passive optical network, Bidirectional signal transmission, Wavelength division multiplexing OCIS codes : (060.0060) Fiber optics and optical communications; (060.2330) Fiber optics communications

\section{INTRODUCTION}

Broadband light source (BLS) seeded optical sources, such as a wavelength-locked Fabry-Perot laser diode (FP-LD) and a reflective semiconductor optical amplifier (RSOA) source, have been widely used for the cost-effective implementation of wavelength-division multiplexed passive optical networks (WDM-PONs) [1-2]. This is because the BLS seeded optical sources could provide colorless operation and a signal modulation and amplification with a single semiconductor device. Recently, the extended-reach PON architectures based on various optical amplifiers and reach extenders have also been proposed for the feasibility demonstration of the cost-effective next-generation optical access networks [3-6]. In the amplified hybrid TDM (time-division multiplexed) / WDM-PON or WDM-PON architectures demonstrated previously, the wavelength-specific optical sources and/or the external modulators were mainly used as WDM optical transmitters $[3,5]$. However, it has been well known that the wavelength-specific optical sources have inventory problems in the implementation of WDM-PON systems [1]. Moreover, these sources are still more expensive than the direct-modulated colorless WDM optical sources based on a BLS. Therefore, we believe that we need to investigate the feasibility of an amplified WDM-PON using only BLS seeded optical sources for the downstream and upstream signals transmission. However, in the amplified PONs using the BLS seeded optical sources, it has been reported that the system's performance could be degraded by the chromatic dispersion and the reflection-induced in-band crosstalk within the transmission fiber [7-8]. In particular, the optical amplifier in-between two fiber links, such as the feeder fiber and the distribution fiber in the PON architectures, could generate the in-band crosstalk components, which in turn would limit the gain of the optical amplifier [9]. In principle, the in-band crosstalk components have the same wavelength as the original signals, thus they could not be simply suppressed with the optical bandpass filter of the optical receiver side. Another issue in the amplified PON using BLS seeded optical sources is that the output power of a BLS for the upstream signal generation (which is located at the central office (CO) generally) should be high enough to overcome the round trip transmission loss. Therefore, the power of optical seed source for the upstream signal generation should be amplified together with the downstream signals in order to resolve the power budget problem in the amplified WDM-PON architectures based on the BLS seeded optical sources. In this paper, we have

\footnotetext{
*Corresponding author: chkim@uos.ac.kr

Color versions of one or more of the figures in this paper are available online.
} 
proposed a novel bidirectional reach extender module for an amplified WDM-PON based on BLS seeded optical sources. The proposed reach extender, implemented simply with two unidirectional optical amplifiers, three band splitters and a circulator, could provide a BLS output for the upstream signal generation as well as the amplification of the downstream and upstream signals. In addition, the proposed reach extender could mitigate the effect of in-band crosstalk on the system's performance. Thus, using the proposed bidirectional reach extender, we have demonstrated the feasibility of a 100-km long BLS based WDM-PON operating at $1.25 \mathrm{~Gb} / \mathrm{s}$.

\section{PROPOSED AMPLIFIED WDM-PON ARCHITECTURE}

Figure 1 shows the proposed scheme of a bidirectional reach extender module which was implemented with two unidirectional optical amplifiers, three band splitters, and a circulator. Here, we assumed that two separate wavelength bands were generally allocated for the downstream and upstream signals transmission in the BLS based WDM-PONs [1-2]. For example, the L- and C-band would be allocated for the downstream and upstream signal transmissions, respectively [1]. In this case, the unidirectional optical amplifier for the downstream signal $\left(\mathrm{G}_{1}\right)$ in our bidirectional reach extender should be implemented with a wideband optical amplifier, which could provide an output spectrum over the C- and L-band. Therefore, this wide optical amplifier for $\mathrm{G}_{1}$ could provide the amplification of L-band downstream signals and the C-band BLS output for the upstream signal generation. One candidate for this wideband optical amplifier in our bidirectional reach extender might be the bismuth-based erbium-doped fiber amplifier (EDFA) [10]. On the other hand, the unidirectional optical amplifier for the upstream signal $\left(\mathrm{G}_{2}\right)$ could be simply implemented with the conventional C-band EDFA. Then, as shown in Fig. 1, two band splitters (1 and 2) were placed at both sides of the bidirec-

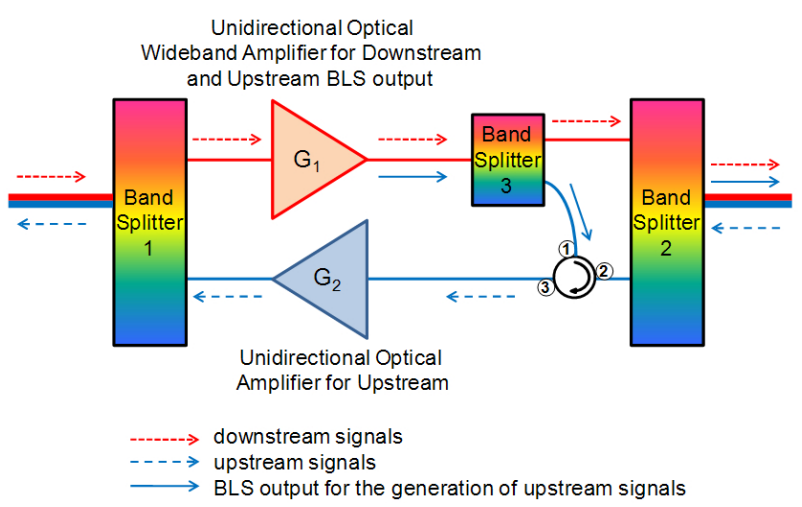

FIG. 1. Proposed bidirectional reach extender module implemented with two unidirectional optical amplifiers, three band splitters, and a circulator. tional reach extender module in order to separate the amplification paths of downstream and upstream signals. These two band splitters could also suppress the effect of unwanted reflected signals from the transmission fiber links. For example, the reflected downstream signals from the right hand side of the module due to the Rayleigh backscattering or a discrete reflectance within a distribution fiber could not be launched into the amplifier $G_{2}$ with the upstream signals because of the pass band characteristics of the band splitter 2. Thus, the proposed bidirectional reach extender could suppress efficiently the effect of in-band crosstalk (mainly generated by a double Rayleigh backscattering) in a bidirectional signal transmission over a single fiber. Therefore, the gains of the unidirectional amplifiers in the proposed bidirectional reach extender would not be limited by the in-band crosstalkinduced penalty [9]. A band splitter 3 was used to separate the output spectrum of the wideband optical amplifier $\left(\mathrm{G}_{1}\right)$ into two parts; one for the wavelength band of downstream signal amplification (i. e. L-band) and the other for the BLS output for the upstream signal generation (i. e. C-band). This separated BLS output was combined again with the amplified downstream signals after passing through a circulator and the band splitter 2, and then transmitted to the optical network units (ONUs) for the upstream signal generation. The generated upstream signals at each ONU could be also amplified in the lower path of the bidirectional reach extender, as shown in Fig. 1.

Figure 2 shows the proposed amplified WDM-PON architecture based on the bidirectional reach extender module. At $\mathrm{CO}$, the downstream signals were generated with a BLS, a 3-port circulator, an arrayed waveguide grating (AWG 1) multiplexer/demultiplexer and an array of RSOAs. A second 3-port circulator was used for the launching of the downstream signals into the feeder fiber as well as the receiving of the upstream signals. Apparently, two 3-port circulators located at $\mathrm{CO}$ could be replaced with a 4-port circulator. We assumed that the effect of chromatic dispersion within the transmission fiber links could be mitigated by using a dispersion compensating fiber (DCF) located at CO. After passing through a DCF and a feeder fiber, the downstream signals could be amplified within the proposed reach extender located at the remote node (RN). The amplified downstream signals together with a BLS output

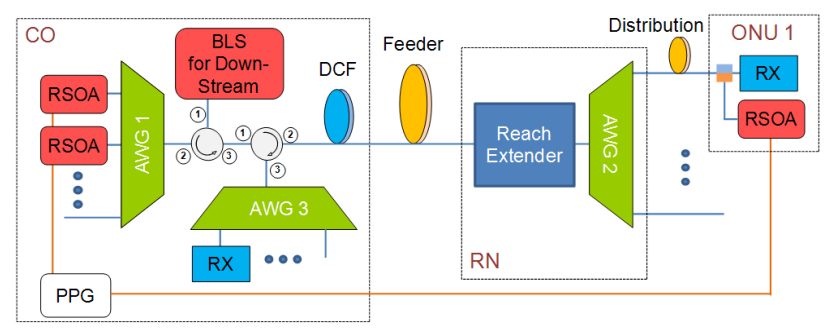

FIG. 2. Proposed amplified WDM-PON architecture based on a novel bidirectional reach extender module and BLS seeded RSOA sources. 
generated within the bidirectional reach extender were transmitted to ONUs through the AWG 2 and the distributed fiber link. As shown in Fig. 2, the upstream signals were generated at each ONU with a RSOA and a BLS output from the bidirectional reach extender. The generated upstream signals were transmitted over the transmission fiber links and amplified $\left(\mathrm{G}_{2}\right)$ at the bidirectional reach extender, and then finally received at $\mathrm{CO}$ after passing through a DCF, a circulator and an AWG 3 .

\section{RESUTS AND DISCUSSION}

For the feasibility demonstration of the amplified WDM-PON using BLS seeded optical sources, the proposed bidirectional reach extender was implemented with two conventional EDFAs consisted of a 980-nm pump LD, a WDM, a length of EDF and two isolators for the amplification of downstream $\left(\mathrm{G}_{1}\right)$ and upstream $\left(\mathrm{G}_{2}\right)$ signals, respectively. The reason why we did not use a wide optical amplifier for $\mathrm{G}_{1}$ was that the wide optical amplifier was not available in our lab at the moment. Therefore, in order to fully utilize the output spectrum of a EDFA, we used the band splitter having two pass bands; one for downstream signals ranging from $1542 \mathrm{~nm}$ to $1558 \mathrm{~nm}$ and the other for the upstream signals ranging from $1522 \mathrm{~nm}$ to $1538 \mathrm{~nm}$. In addition, the distribution fiber was temporarily placed in-between the bidirectional reach extender and an AWG 2 to evaluate the effect of bidirectional signal transmission over a single distribution fiber without using a cyclic AWG [1]. For the performance evaluation of the system, we first generated two downstream signals operating at $1549.3 \mathrm{~nm}$ and 1550.9 $\mathrm{nm}$ and an upstream signal operating at $1531.1 \mathrm{~nm}$ using three RSOAs. Both AWG 1 and 2 had a 3-dB bandwidth of $0.4 \mathrm{~nm}$, and they were used to generate the spectrally-sliced BLS outputs for the downstream and upstream signals, respectively. The powers of spectrally-sliced BLS outputs into three RSOAs were set to be $-15 \mathrm{dBm}$. Each RSOA was directly-modulated with a $1.25 \mathrm{~Gb} / \mathrm{s}$ nonreturn-to-zero
(NRZ) pseudorandom pattern of length $2^{31}$-1. In our experimental demonstration, a DCF module having an insertion loss of $8 \mathrm{~dB}$ and a dispersion of -1350 ps/nm (@1550 nm)was used to compensate the chromatic dispersion of the transmission fiber. However, the total length of our transmission fiber link was about $100-\mathrm{km}$, which consisted of a $75-\mathrm{km}$ single-mode fiber (SMF) feeder fiber and a $25-\mathrm{km}$ SMF distribution fiber. Therefore, the residual dispersions of the downstream (@1549.3 nm) and upstream (@1531.1 nm) signals were measured to be $383 \mathrm{ps} / \mathrm{nm}$ and $320 \mathrm{ps} / \mathrm{nm}$, respectively.

Figure 3 shows the optical spectra of the downstream signals measured directly at the input and output of the proposed bidirectional reach extender. From the results, we confirmed that the gain of downstream signals in our reach extender could be increased to $\sim 30 \mathrm{~dB}$. In principle, the gain of the upstream signal could also be set to $\sim 30 \mathrm{~dB}$ by adjusting the pump power of EDFA $\left(\mathrm{G}_{2}\right)$. We also measured the optical spectra in-between the bidirectional reach extender and the distribution fiber using a $2 \times 2 \quad 1 \%$ tap in both directions. Fig. 4(a) shows the optical spectrum of amplified downstream signals with a BLS output for the

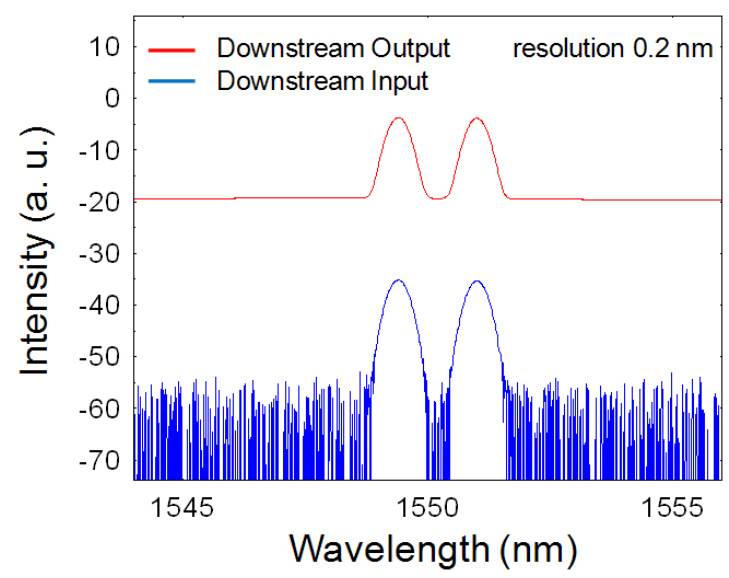

FIG. 3. Optical spectra of downstream signals measured at input and output of a proposed bidirectional reach extender.

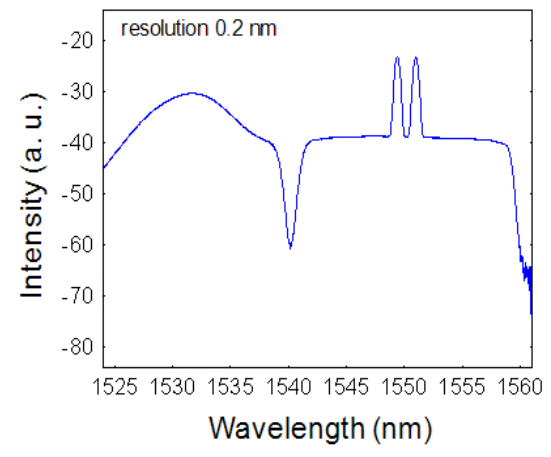

(a)

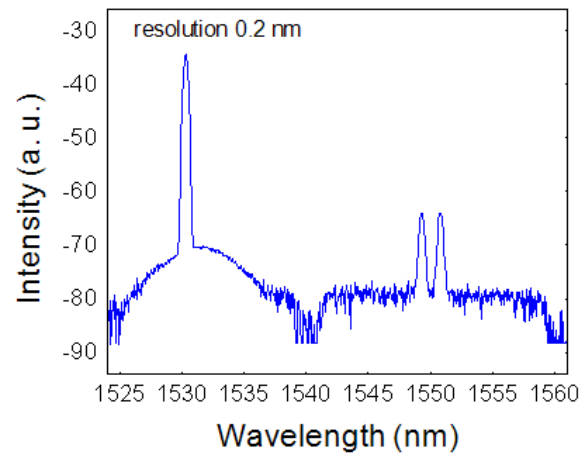

(b)

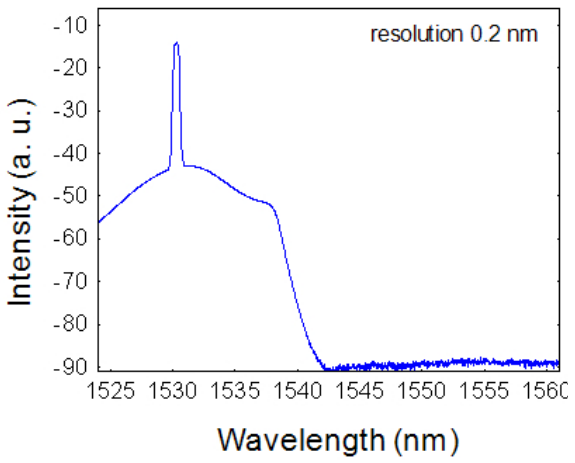

(c)

FIG. 4. Optical spectra measured (a) in-between the proposed reach extender and the distribution fiber in downstream direction (output downstream signal with a BLS output), (b) in upstream direction (input upstream signal with the reflected downstream signals) and (c) in-between the feeder fiber and the proposed reach extender (output upstream signal). 
upstream signal generation. The input upstream signal of our bidirectional reach extender was also measured with the reflected downstream signals generated from a Rayleigh backscattering within the distribution fiber, as shown in Fig. 4(b). However, these reflected downstream signals would be suppressed with the band splitter 2 of Fig. 1. To confirm this, the output spectrum of the upstream signal was measured in-between the bidirectional reach extender and the feeder fiber with a $1 \%$ tap, as shown in Fig. 4(c). From the spectrum, we found that the reflected downstream signals were suppressed efficiently with the band splitters, as expected. We also confirmed that, the reflected upstream signal from the feeder fiber was suppressed with our bidirectional reach extender, as can be seen in Fig 4(a). Thus, we confirmed that our bidirectional reach extender would not generate double Rayleigh backscattering induced in-band crosstalk components, since the reflected downstream and upstream signals would not be allowed to be amplified in the lower and upper paths of our module, respectively.

In order to analyze the power budget in the proposed amplified WDM-PON, we measured the power levels of the downstream and upstream signals at various points with a tunable bandpass filter and an optical power meter, as shown in Table 1. From the results, we found that the power margins in both directions were good enough for error-free signal transmission by considering the sensitivity of our receiver. In addition, the operating gains of the proposed reach extender were measured to be $31.5 \mathrm{~dB}$ for the downstream signal and $19.4 \mathrm{~dB}$ for the upstream signal. The noise figure of the bidirectional reach extender was also measured to be $\sim 5.5 \mathrm{~dB}$ in both directions.

Figure $5(\mathrm{a})$ shows the eye diagrams of a $1.25 \mathrm{~Gb} / \mathrm{s}$

TABLE 1. Power levels of downstream and upstream signals measured at various points in the implemented amplified WDM-PON

\begin{tabular}{c|c|c|c|c|c}
\hline \hline $\begin{array}{c}\text { Location } \\
\text { (in-between) }\end{array}$ & $\begin{array}{c}\text { A Second Circulator } \\
\text { and DCF }\end{array}$ & DCF and Feeder & $\begin{array}{c}\text { Feeder and Reach } \\
\text { Extender }\end{array}$ & $\begin{array}{c}\text { Reach Extender and } \\
\text { Distribution }\end{array}$ & $\begin{array}{c}\text { Rx Input or RSOA } \\
\text { Output }\end{array}$ \\
\hline $\begin{array}{c}\text { Downstream } \\
(\mathrm{dBm} / \mathrm{ch})\end{array}$ & -7.1 & -15.1 & -32.3 & -0.8 & -9 \\
\hline $\begin{array}{c}\text { Upstream } \\
(\mathrm{dBm} / \mathrm{ch})\end{array}$ & -19.6 & -11.2 & 6.3 & -13.1 & -4.6 \\
\hline
\end{tabular}
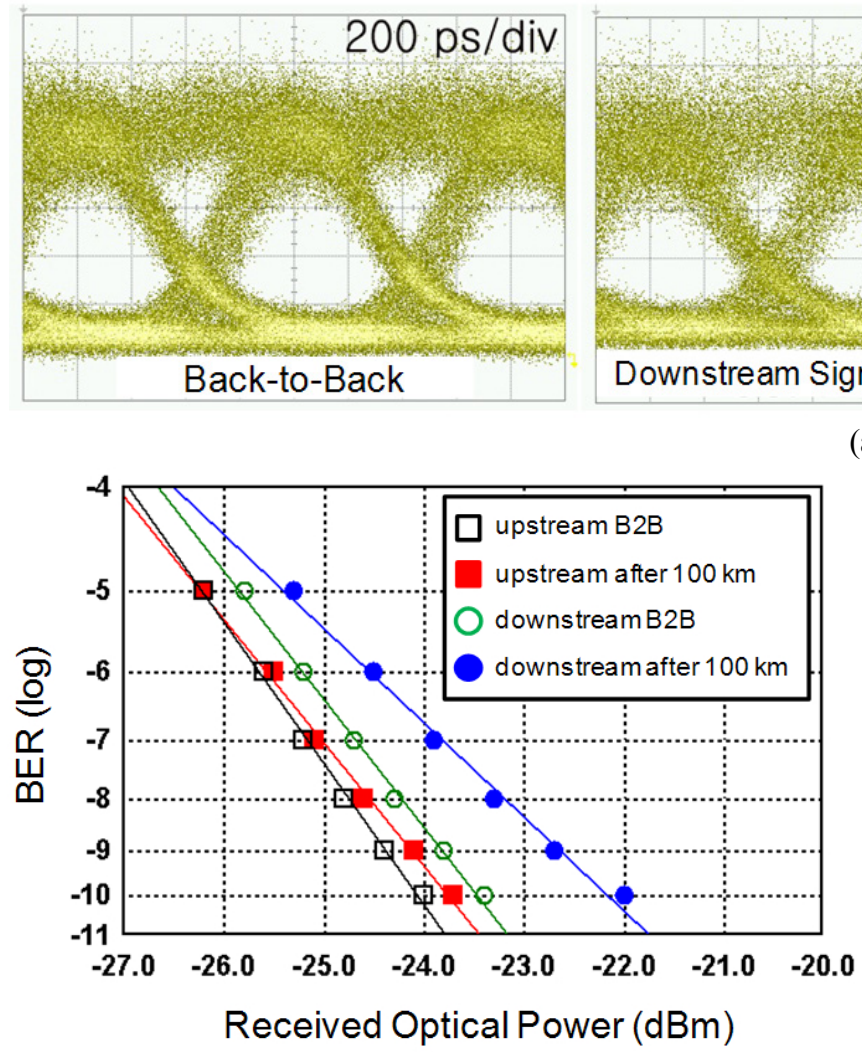

(b)
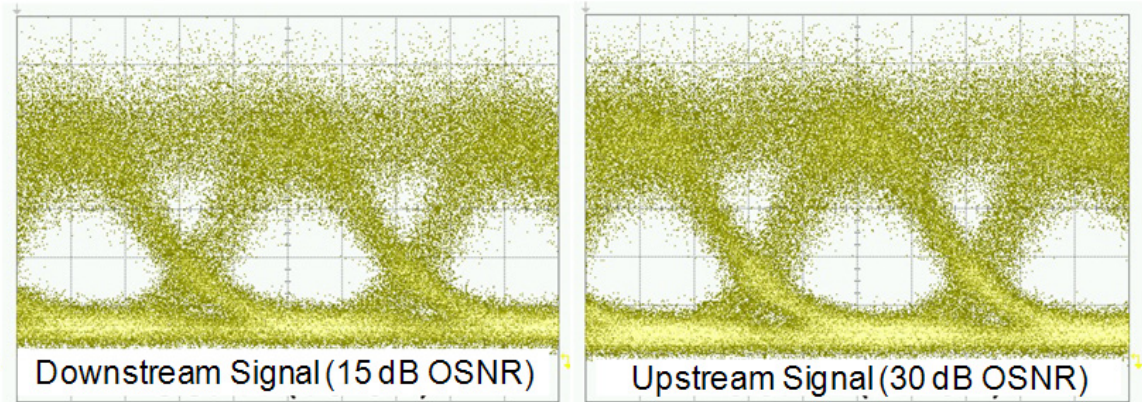

(a)

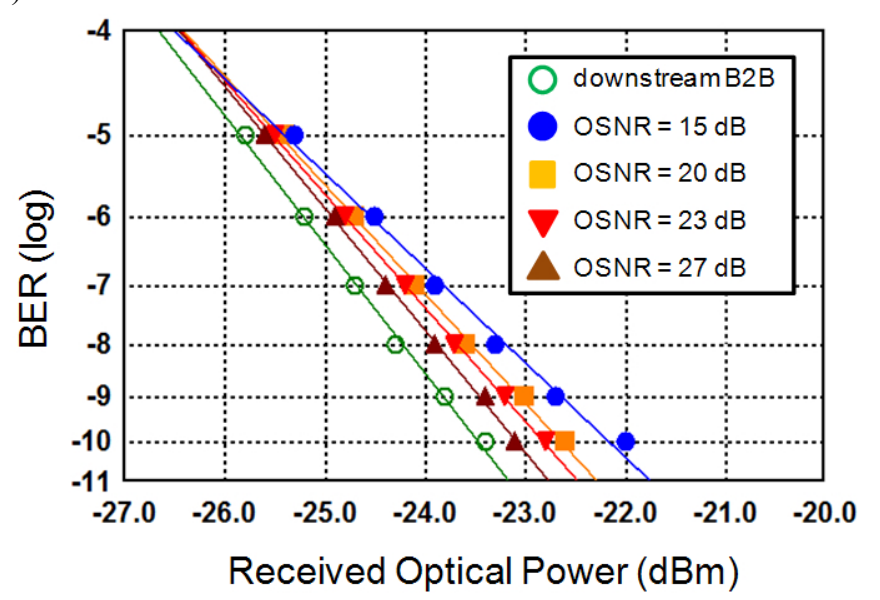

(c)

FIG. 5. (a) Measured eye diagrams and (b) BER curves of both downstream (@1549.3 nm) and upstream(@1531.1 nm) signals, and (c) measured BER curves of downstream signal as a function of OSNR. 
downstream signal (@1549.3 nm) and an upstream signal (@1531.1 nm) measured after a 100-km SMF transmission. For comparison, the eye diagram of the downstream signal in a back-to-back configuration was also measured as shown in Fig. 5(a). In the back-to-back configuration, the extinction ratio of BLS seeded RSOA source was measured to be $\sim 10 \mathrm{~dB}$. The wide open eyes for error-free signal transmission were obtained in both the back-to-back configuration and the $100-\mathrm{km}$ long system. After the $100-\mathrm{km}$ SMF transmission, the optical signal-to-noise ratio (OSNR) of a downstream signal and an upstream signal were measured to be $\sim 15 \mathrm{~dB}$ and $\sim 30 \mathrm{~dB}$ (@0.1 nm resolution), respectively. The low OSNR of the downstream signal was mainly due to the high insertion loss of a DCF as well as to the transmission loss of feeder fiber. The bit-error-rate (BER) performances of both downstream and upstream signals were also measured in a back-to-back and after a $100-\mathrm{km}$ signal transmission, as shown in Fig. 5(b). For an upstream signal transmission, a $0.3-\mathrm{dB}$ penalty at a BER of $10^{-9}$ was observed because of a residual chromatic dispersion of 320 $\mathrm{ps} / \mathrm{nm}$ [7]. For the case of downstream signal transmission, the power penalty was increased to $\sim 1.3 \mathrm{~dB}$. This is mainly because of a low OSNR of $\sim 15 \mathrm{~dB}$ and a residual dispersion of $383 \mathrm{ps} / \mathrm{nm}$. However, no BER floors due to the in-band crosstalk in the bidirectional signal transmission over a single fiber were observed even with the high gain $(\sim 30$ $\mathrm{dB}$ ) of the proposed bidirectional reach extender [9]. In order to investigate the effect of OSNR on the system's performance, we measured the BER curves of the downstream signal as a function of OSNR, as shown in Fig. 5(c). For this measurement, the launched power of the downstream signal into the feeder was adjusted by controlling the gain of another EDFA located at CO. As it can be seen in Fig. $5(\mathrm{c})$, the power penalty of the downstream signal could be reduced to $\sim 0.4 \mathrm{~dB}$ with an OSNR of $27 \mathrm{~dB}$. From the results, we confirmed that the error-free $1.25 \mathrm{~Gb} / \mathrm{s}$ signal transmission over a $100-\mathrm{km}$ long SMF could be achieved in an amplified bidirectional WDM-PON based on BLS seeded RSOA sources and the proposed reach extender. In addition, we believe that the power penalty induced by a low OSNR of downstream signal could be improved by using an alternative dispersion compensation technique, such as an electrical dispersion compensation module or a low-loss fiber Bragg grating.

\section{SUMMARY}

We have demonstrated the feasibility of an amplified bidirectional WDM-PON based on BLS seeded RSOA sources and the proposed bidirectional reach extender. The proposed bidirectional reach extender module could provide a BLS output for the upstream signal generation as well as the high $(>30 \mathrm{~dB})$ amplifier gain for both downstream and upstream signals without in-band crosstalk induced penalty. An error-free $1.25 \mathrm{~Gb} / \mathrm{s}$ signal transmission over a $100-\mathrm{km}$ long SMF was achieved in an amplified WDM-PON based on BLS seeded RSOA sources. From the results, we believe that the proposed bidirectional reach extender would be well suited for the use in the implementation of cost-effective amplified WDM-PONs.

\section{ACKNOWLEDGMENT}

This work was supported by Basic Science Research Program through the National Research Foundation of Korea (NRF) funded by the Ministry of Education, Science and Technology (MEST) (NRF-2008-331-D00375).

\section{REFERENCES}

1. C.-H. Lee and S.-G. Mun, "WDM-PON based on wavelengthlocked Fabry-Perot LDs," J. Opt. Soc. Korea 12, 326-336 (2008).

2. B. W. Kim, "RSOA-based wavelength-reuse gigabit WDM-PON," J. Opt. Soc. Korea 12, 337-345 (2008).

3. R. P. Davey, P. Healey, I. Hope, P. Watkinson, D. B. Payne, O. Marmur, J. Ruhmann, and Y. Zuiderveld, "DWDM reach extension of a GPON to $135 \mathrm{~km}$," J. Lightwave Technol. 24, 29-31 (2006).

4. I. T. Monroy, R. Kjaer, B. Palsdottir, A. M. J. Koonen, and P. Jeppesen, " $10 \mathrm{~Gb} / \mathrm{s}$ bidirectional single fibre long reach PON link with distributed Raman amplification," in Proc. Eur. Conf. Optical Communication 2006 (Cannes, France, Sep. 2006), We3.P.166.

5. H. H. Lee, K. C. Reichmann, P. P. Iannone, X. Zhou, and B. Palsdottir, "A hybrid-amplified PON with 75-nm downstream band-with, $60 \mathrm{~km}$ reach, 1:64 split and multiple video services," in Proc. OFC/NFOEC 2007 (Anaheim, CA, USA, Mar. 2007), OWL2.

6. C. H. Kim, J. H. Lee, and K. Lee, "Analysis of maximum reach in WDM PON architecture based on distributed Raman amplification and pump recycling technique," Opt. Express 15, 14942-14947 (2007).

7. C. H. Kim, J. H. Lee, D. K. Jung, Y.-G. Han, and S. B. Lee, "Performance comparison of directly-modulated, wavelengthlocked Fabry-Perot laser diode and EAM-modulated spectrumsliced ASE source for $1.25 \mathrm{~Gb} / \mathrm{s}$ WDM-PON," in Proc. OFC/NFOEC 2007 (Anaheim, CA, USA, Mar. 2007), JWA82.

8. C. H. Kim, K. Lee, and S. B. Lee, "Effects of in-band crosstalk in wavelength-locked Fabry-Perot laser diode based WDM PONs," IEEE Photon. Technol. Lett. 21, 596-598 (2009).

9. B.-W. Kang and C. H. Kim, "Performance evaluation of bidirectional optical amplifiers for amplified passive optical network based on broadband light source seeded optical sources," J. Opt. Soc. Korea 15, 4-8 (2011).

10. B.-O. Guan, H.-W. Tam, S.-Y. Liu, P. K. A. Wai, and N. Sugimoto, "Ultra-wideband bismuth-based EDFA for DWDM systems," in Proc. the Sixth Chinese Optoelectronics Symposium (Hong Kong University of Science and Technology, Hong Kong, Sep. 2003), pp. 147-149. 